doi:10.1038/pr.2017.214

\section{Early Career Investigator}

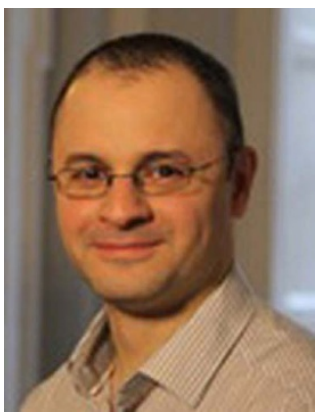

Congratulations to Afif EL-Khuffash, $\mathrm{MB}, \mathrm{BCh}, \mathrm{BAO}, \mathrm{BA}(\mathrm{SCi})$, FRCPI, MD, $D C E$, the Early Career Investigator for November! Read his Commentary to discover how this son of two Kuwaiti pediatricians wound up as a productive scientist and pediatric cardiologist. His article in this issue explores the feasibility and reliability of noninvasive cardiac output and cerebral perfusion monitoring in infants with encephalopathy. See pages 722 and 789

\section{Enhancing clinical trial development for pediatric kidney disease}

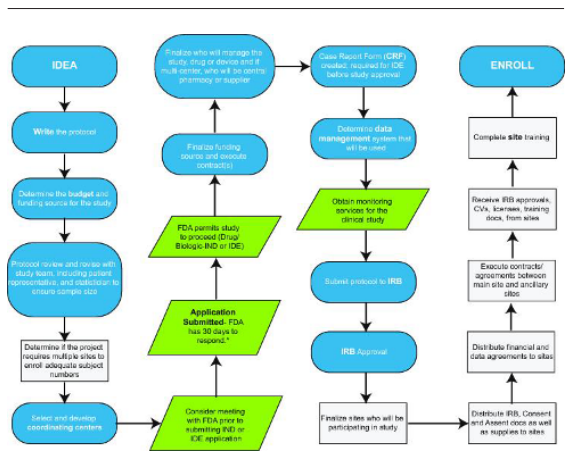

In their Review, Schnaper et al. describe the rationale, structure, and function of the American Society of Pediatric Nephrology Therapeutics Development Committee (TDC), its goals in facilitating clinical trials within pediatric nephrology and its progress to date. The experience of the TDC may guide other subspecialties in developing strategies to accomplish similar goals. See page 727

\section{Polymorphisms in CYP2C9 are associated with indomethacin's effectiveness}

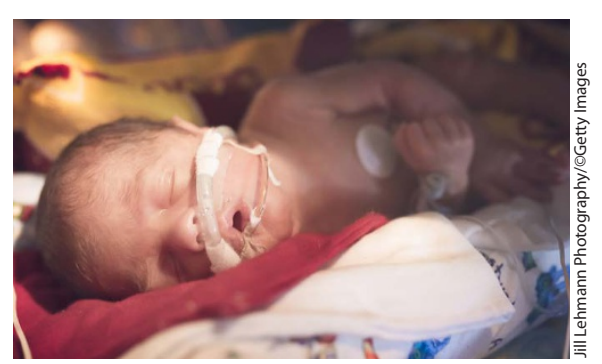

Indomethacin is a typical pharmacologic intervention for the closure of patent ductus arteriosus. However, the response to indomethacin is heritable. Smith et al. examined six polymorphisms in CYP2C9 to explain the variability in the efficacy of this drug. See page 776

\section{Serum levels of prolactin and growth hormone in the neonate}

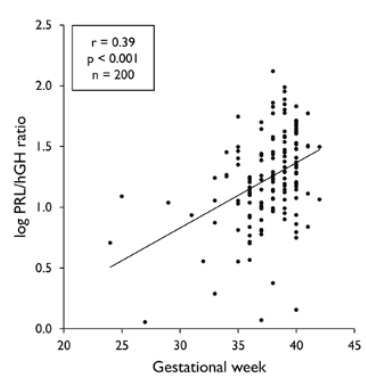

Daliot et al. explored the relationship between two hormones that are secreted by the same somatomammotrophic cells within the first month after birth. The ratio of the two hormones was found to be strongly influenced by gestational age. See page 796

\section{Long-term effects of adenotonsillectomy on serum-specific IgE}

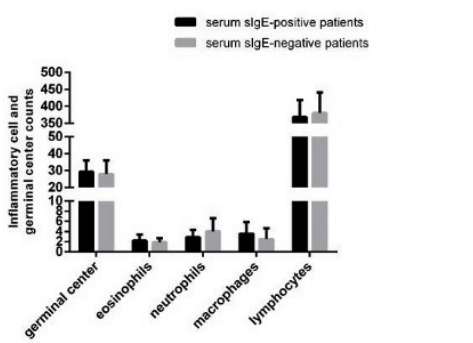

Song et al. hypothesized that systemic atopic status is not altered after adenotonsillectomy (AT) in children. They followed 25 children who had undergone AT and 23 age-matched controls for 4 years. The results indicated that AT does not affect pediatric atopic status. See page 801

\section{No sex difference in cerebrovascular functions following epileptic seizures}

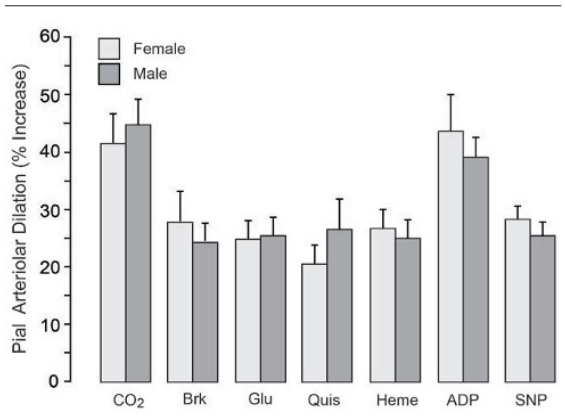

Liu et al. examined sex differences in the responses of pial arterioles to endothelium-, astrocyte-, and vascular smooth muscle-dependent vasodilators in pigs following bicuculline-induced seizures. The authors found no sex differences, but they describe prolonged cerebral vascular dysfunction that was effectively prevented by a carbon monoxide-releasing molecule. See page 881

\section{Pediatric clinical trial feasibility and Bayesian statistics}

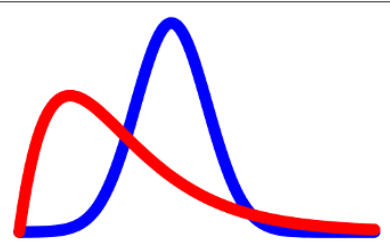

Enrolling a sufficient number of patients to reach significance in pediatric clinical trials can be a difficult hurdle. The use of Bayesian statistics in trial design may make it possible to reduce the sample size, increasing the feasibility of pediatric clinical trials. See page 814 